\title{
The Concept of $q$-Cycle and Applications
}

\author{
Umarin Pintoptang, ${ }^{1,2}$ Suton Tadee, ${ }^{3}$ and Vichian Laohakosol ${ }^{4}$ \\ ${ }^{1}$ Department of Mathematics, Faculty of Science, Naresuan University, Phitsanulok 65000, Thailand \\ ${ }^{2}$ Centre of Excellence in Mathematics (CHE), Si Ayutthaya Road, Bangkok 10400, Thailand \\ ${ }^{3}$ Department of Mathematics and Statistics, Faculty of Science and Technology, Thepsatri Rajabhat University, \\ Lopburi 15000, Thailand \\ ${ }^{4}$ Department of Mathematics, Faculty of Science, Kasetsart University, Bangkok 10900, Thailand
}

Correspondence should be addressed to Vichian Laohakosol; fscivil@ku.ac.th

Received 7 October 2013; Revised 9 December 2013; Accepted 28 December 2013; Published 26 February 2014

Academic Editor: Franck Petit

Copyright (C) 2014 Umarin Pintoptang et al. This is an open access article distributed under the Creative Commons Attribution License, which permits unrestricted use, distribution, and reproduction in any medium, provided the original work is properly cited.

The concept of $q$-cycle is investigated for its properties and applications. Connections with irreducible polynomials over a finite field are established with emphases on the notions of order and degree. The results are applied to deduce new results about primitive and self-reciprocal polynomials.

\section{Introduction}

Let $\mathbb{F}_{q}$ denote the finite field of $q$ elements and let $N \in \mathbb{N}$, $\operatorname{gcd}(q, N)=1$. Let $a_{0}, a_{1}, \ldots, a_{\ell-1}$ be $\ell$ distinct numbers chosen from $\mathbb{Z}_{N}:=\{0,1, \ldots, N-1\}$. If

$$
\begin{gathered}
a_{i} q \equiv a_{i+1} \bmod N \quad(i=0,1, \ldots, \ell-2), \\
\left(a_{0} q^{\ell}\right) \equiv a_{\ell-1} q \equiv a_{0} \bmod N
\end{gathered}
$$

then we say that $\left(a_{0}, a_{1}, \ldots, a_{\ell-1}\right)$ forms a $q$-cycle $\bmod N$ with leading element $a_{0}$, abbreviated by $q\left(a_{0}\right)$-cycle $(N)$ or $q$ $\operatorname{cycle}(N)$ when the leading element in the cycle is immaterial, and call $\ell$ the length of this $q\left(a_{0}\right)$-cycle $(N)$. The notion of $q$ cycles was introduced by Wan in his book [1, page 203]. Since $q^{o_{N}(q)} \equiv 1 \bmod N$, where $o_{N}(q)$ is the order of $q$ in $\mathbb{Z}_{N}^{*}:=$ $\mathbb{Z}_{N} \backslash\{0\}$ (the multiplicative group of nonzero integers modulo $N)$, it clearly follows that each $q(a)-\operatorname{cycle}(N)$ always has a unique length $\ell$ which is the least positive integer $\ell$ for which $a \equiv a q^{\ell} \bmod N$. Observe that $q$-cycles are nothing but $\mathbb{Z}$ orbits $q^{\mathbb{Z}} a, a \in \mathbb{Z}_{N}$; that is, $1 \in \mathbb{Z}$ acts on $\mathbb{Z}_{N}$ by multiplication with $q$. The concept of $q$-cycles is important because of the following connections with irreducible polynomials.

(A) (see [1, Theorem 9.11]) Let $\alpha$ be a primitive $N$ th root of unity (if the order of $q$ in $\mathbb{Z}_{N}^{*}$ is $m$, then there exists a primitive
$N$ th root of unity in $\left.\mathbb{F}_{q^{m}}\right)$. If $\left(a_{0}, a_{1}, \ldots, a_{\ell-1}\right)$ is a $q$-cycle $(N)$, then

$$
h(x)=\left(x-\alpha^{a_{0}}\right)\left(x-\alpha^{a_{1}}\right) \cdots\left(x-\alpha^{a_{\ell-1}}\right)
$$

is a monic irreducible factor of $x^{N}-1$ in $\mathbb{F}_{q}[x]$. Conversely, if $h(x)$ is a monic irreducible factor of $x^{N}-1$ in $\mathbb{F}_{q}[x]$, then all the roots of $h(x)$ are powers of $\alpha$ whose exponents form a $q$-cycle $(N)$. We henceforth refer to these two facts as the $\alpha$ correspondence.

(B) (see [1, Corollary 9.12]) The number of distinct irreducible polynomials dividing $x^{N}-1$ in $\mathbb{F}_{q}[x]$ is equal to the number of $q$-cycles $(N)$ formed with the leading $N$ elements taken from $\{0,1, \ldots, N-1\}$.

Our objectives here are to illustrate the versatility of $q$ cycles by using them to prove new results about irreducible polynomials. General properties of $q$-cycles are given in the next section; specific details in two special cases corresponding to $N=q^{n}-1, q^{m}+1$ are worked out for applications to primitive and self-reciprocal polynomials in the last section. Section 3 deals with results about the order of a polynomial, while Section 4 does the same for the degree of a polynomial. Section 5 shows that knowing a $q$-cycle is equivalent to knowing all coefficients of the corresponding 
polynomial (2). The last section provides applications of $q$ cycles to primitive and self-reciprocal polynomials.

Notation and Terminology. Throughout, we fix the following symbols and their meanings.

(i) $p$ is a fixed prime, $q$ is a power of $p$, and $\mathbb{F}_{q}$ is the finite field with $q$ elements.

(ii) $N$ is a fixed positive integer such that $\operatorname{gcd}(q, N)=1$.

(iii) $d:=d(N)$ is the number of (positive integer) divisors of $N$.

(iv) $N=N_{1}>N_{2}>\cdots>N_{d}=1$ (all divisors of $N$ ).

(v) For $n \in \mathbb{N}$, the Möbius function $\mu$ is defined by

$$
\mu(n)= \begin{cases}1, & \text { if } n=1, \\ (-1)^{k}, & \text { if } n \text { is a squarefree product of } \\ & k \text { distinct primes, } \\ 0, & \text { if } n \text { is not squarefree. }\end{cases}
$$

(vi) For $n \in \mathbb{N}$, the Euler's function $\varphi(n)$ is the number of integers $k \in\{1,2, \ldots, n\}$ with $\operatorname{gcd}(k, n)=1$.

(vii) For $M \in \mathbb{N}$, denote by $o_{M}(q)$ the order of $q$ in $\mathbb{Z}_{M}^{*}:=$ $\mathbb{Z}_{M} \backslash\{0\}$; that is,

$$
q^{o_{\mathrm{M}}(q)} \equiv 1 \bmod M
$$

but $q^{i} \not \equiv 1 \bmod M \quad \forall i \in \mathbb{N}, i<o_{M}(q)$.

(viii) For $r \in\{1,2, \ldots, d\}$, set $\mathscr{C}_{N_{r}}=\left\{q\right.$-cycles $\left.\left(N_{r}\right)\right\}$, the set of all $q$-cycles $\bmod N_{r}$.

\section{Properties of $q$-Cycles}

Let $\left(a_{0}, a_{1}, \ldots, a_{\ell-1}\right)$ be a $q\left(a_{0}\right)$-cycle $(N)$. It is easy to see that $\mathbb{Z}_{N}$ can be decomposed into a finite union of disjoint $q^{-}$ $\operatorname{cycles}(N)$, namely,

$$
\mathbb{Z}_{N}=(0) \cup\left(1, q, q^{2}, \ldots, q^{o_{N}(q)-1}\right) \bigcup_{a \in \mathbb{Z}_{N} \backslash\{0,1\}}\left(a, a q, a q^{2}, \ldots\right) .
$$

Since $q^{o_{N}(q)} \equiv 1 \bmod N$ and $a q^{o_{N}(q)} \equiv a \bmod N$ for all $a \in \mathbb{Z}_{N}$, each $q$-cycle $(N)$ is of length $\leq o_{N}(q)$ with the one containing 1 having the largest length. We collect in our first theorem further properties of $q$-cycles, whose straightforward proofs are omitted.

Theorem 1. Let $a, b \in \mathbb{Z}_{N}, N_{0}:=\mathbb{N} \cup\{0\}$.

(i) The element $b \in q(a)$-cycle $(N)$ if and only if $b \equiv$ $a q^{m} \bmod N$ for some $m \in \mathbb{N}_{0}$.

(ii) Two q-cycles, $q(a)$-cycle $(N)$ and $q(b)$-cycle $(N)$, are identical if and only if $b \in q(a)$-cycle $(N)$.

(iii) For $b \in \mathbb{Z}_{N}^{*}$, each $q(b)$-cycle $(N)$ has length $\ell$ if and only if $N / \operatorname{gcd}(b, N)$ divides $q^{\ell}-1$ but does not divide $q-$ $1, q^{2}-1, \ldots, q^{\ell-1}-1$ (if $\ell=1$, only the first divisibility needs to be checked). The $q(0)$-cycle $(N)$ has length 1 . (iv) The length of each $q$-cycle $(N)$ divides $o_{N}(q)$.

(v) If $b \in \mathbb{Z}_{N}$ is such that $\operatorname{gcd}(b, N)=N_{r}$, then the $q(b)$ cycle $(N)$ has length $o_{N / N_{r}}(q)$, and there are altogether $\varphi\left(N / N_{r}\right) / o_{N / N_{r}}(q)$ distinct such q-cycles. In particular, if $b \in \mathbb{Z}_{N}$ is such that $\operatorname{gcd}(b, N)=1$, then the $q(b)$ cycle $(N)$ has length $o_{N}(q)$, and there are altogether $\varphi(N) / o_{N}(q)$ distinct $q$-cycles with length $o_{N}(q)$.

(vi) The length of any $q$-cycle $(N)$ is of the form $o_{N_{r}}(q)$ for some $r \in\{1, \ldots, d\}$.

(vii) The number of $q$-cycles $(N)$ of length $\ell$ is $(1 / \ell) \sum_{1 \leq r \leq d, o_{N_{r}}}(q)=\ell \varphi\left(N_{r}\right)$.

(viii) The total number of $q$-cycles $(N)$ is $\left|\mathscr{C}_{N}\right|=\sum_{1 \leq r \leq d}$ $\left(\varphi\left(N_{r}\right) / o_{N_{r}}(q)\right)(|\cdot|$ signifies the number of elements in the set).

2.1. Two Special Cases. There are two particular cases $N=$ $q^{n}-1$ and $N=q^{m}+1$, which are closely related to primitive and self-reciprocal and will be needed in the last section.

2.1.1. The Case $N=q^{n}-1$. When $N=q^{n}-1(n \in \mathbb{N})$, more precise information is now derived.

Theorem 2. Let $n \in \mathbb{N}$. Then

(i) $o_{q^{n}-1}(q)=n$;

(ii) $n$ is the largest length among $q$-cycles $\left(q^{n}-1\right)$ and any other length of $q$-cycles $\left(q^{n}-1\right)$ divides $n$;

(iii) the number of $q$-cycles $\left(q^{n}-1\right)$ having length $\ell$ is equal to

$$
\frac{1}{\ell} \sum_{\substack{1 \leq r \leq d \\ o_{N_{r}}(q)=\ell}} \varphi\left(N_{r}\right)= \begin{cases}q-1 & \text { if } \ell=1 \\ \frac{1}{\ell} \sum_{k \mid \ell} \mu(k) q^{\ell / k} & \text { if } \ell>1 .\end{cases}
$$

Proof. Assertions (i) and (ii) are immediate from the definition and Theorems 1(iii)-(iv). To verify (iii), observe that if $\ell=1$, then it is easily checked that all the $q$-cycles $\left(q^{n}-1\right)$ of length 1 are those starting with $0, A:=q^{n-1}+q^{n-2}+$ $\cdots+q+1,2 A, \ldots,(q-2) A$ whose total number is $q-1$. Assume now that $\ell>1$ and that the assertion holds up to $\ell-1$. From Theorem 1 (vii), the number of $q$-cycles $\left(q^{n}-1\right)$ having length $\ell$ is $(1 / \ell) \sum_{1 \leq r \leq d, o_{N_{r}}(q)=\ell} \varphi\left(N_{r}\right)$. Thus, to prove the desired result, we only need to verify that

$$
\sum_{\substack{1 \leq r \leq d \\ o_{N_{r}}(q)=\ell}} \varphi\left(N_{r}\right)=\sum_{k \mid \ell} \mu(k) q^{\ell / k}
$$


Consider

$$
\begin{aligned}
\sum_{\substack{1 \leq r \leq d \\
o_{N_{r}}(q)=\ell}} \varphi\left(N_{r}\right)= & \sum_{\substack{N_{r}\left|\left(q^{\ell}-1\right) \\
N_{r}+\left(q^{s}-1\right) \forall s\right| \ell, 1 \leq s<\ell}} \varphi\left(N_{r}\right) \\
& =\sum_{N_{r} \mid\left(q^{\ell}-1\right)} \varphi\left(N_{r}\right)-\sum_{\substack{N_{r}\left|\left(q^{s}-1\right) \\
\forall s\right| \ell, 1 \leq s<\ell}} \varphi\left(N_{r}\right) \\
& =q^{\ell}-1-\sum_{\substack{N_{r}\left|\left(q^{s}-1\right) \\
\forall s\right| \ell, 1 \leq s<\ell}} \varphi\left(N_{r}\right) .
\end{aligned}
$$

Using the induction hypothesis for the second term on the right-hand side of (8) with an extra -1 to take care of the case $\ell=1$, we get

$$
\begin{aligned}
\sum_{\substack{N_{r}\left|\left(q^{s}-1\right) \\
\forall s\right| \ell, 1 \leq s<\ell}} \varphi\left(N_{r}\right) & =\sum_{\substack{s \mid \ell \\
1 \leq s<\ell}}\left(\sum_{t \mid s} \mu(t) q^{s / t}\right)-1 \\
& =\sum_{\substack{k \mid \ell \\
1 \leq k<\ell}} q^{k} \sum_{\substack{i k \mid \ell \\
1 \leq i k<\ell}} \mu(i)-1 \\
& =\sum_{\substack{k \mid \ell \\
1 \leq k<\ell}} q^{k}\left(\sum_{i \mid(\ell / k)} \mu(i)-\mu\left(\frac{\ell}{k}\right)\right)-1 \\
& =-\sum_{\substack{k \mid \ell \\
1 \leq k<\ell}} q^{k} \mu\left(\frac{\ell}{k}\right)-1 \\
& =-\sum_{\substack{k \mid \ell \\
1 \leq k<\ell}} \mu(k) q^{\ell / k}-1 .
\end{aligned}
$$

The result now follows by substituting this into (8).

Implicit in the proof of Theorem 2 is the following nice identity.

Corollary 3. With the same notation as in Theorem 2, one has

$$
\sum_{\substack{1 \leq r \leq d \\ o_{N_{r}}(q)=\ell}} \varphi\left(N_{r}\right)=\sum_{d \mid \ell} \mu(d) q^{\ell / d}-\left\lfloor\frac{1}{\ell}\right\rfloor .
$$

2.1.2. The Case $N=q^{m}+1$. When $N=q^{m}+1(m \in \mathbb{N})$, more precise information about $q$-cycles is now derived.

Theorem 4. Let $m \in \mathbb{N}$. Then

(i) $o_{q^{m}+1}(q)=2 m$;

(ii) $2 m$ is the largest length among $q$-cycles $\left(q^{m}+1\right)$ and any other length divides $2 m$;

(iii) the number of $q$-cycles $\left(q^{m}+1\right)$ having length $\ell$ is $(1 / \ell) \sum_{1 \leq r \leq d, o_{N_{r}}(q)=\ell} \varphi\left(N_{r}\right)$.

Proof. Since $q^{2 m} \equiv 1 \bmod q^{m}+1$, we have $o_{q^{m}+1}(q) \mid 2 m$. Assertion (i) thus follows from the observation that $o_{q^{m}+1}(q)>m$. Assertions (ii) and (iii) are direct consequences of Theorems 1(iii)-(vii).
In [2], Yucas and Mullen introduced the following set:

$$
\begin{aligned}
D_{m}:= & \left\{n \in \mathbb{N} ; n \mid\left(q^{m}+1\right),\right. \\
& \left.n+\left(q^{k}+1\right), k \in\{0,1, \ldots, m-1\}\right\} .
\end{aligned}
$$

We show next that elements in this set can also be described through order.

Lemma 5. Let $m, n(>2) \in \mathbb{N}$. Then

$n \in D_{m} \Leftrightarrow$ the multiplicative order of $q \bmod n$ is $2 m$, that is, $o_{n}(q)=2 m$, and there is a positive integer $t$ dividing $m$ such that $n \mid\left(q^{t}+1\right)$.

Proof. If $n \in D_{m}$, then $n \mid\left(q^{2 m}-1\right)$. If $n \mid\left(q^{s}-1\right)(s \in \mathbb{N})$, then [2, Proposition 1] tells us that $s$ must be an even multiple of $m$. Thus, $o_{n}(q)=2 m$. Conversely, assume that $o_{n}(q)=2 m$ and $t$ is the least positive integer for which $n \mid\left(q^{t}+1\right)$. Thus, [2, Proposition 1] tells us that $2 m$ is an even multiple of $t$, and so $t \mid m$. If $t<m$, the divisibility $n \mid\left(q^{2 t}-1\right)$ contradicts $o_{n}(q)=2 m$. Hence, $t=m$.

The next proposition, which is of independent interest, connects the sum in Theorem 4(iii) with one involving the Möbius function.

Proposition 6. One has

$$
\begin{array}{r}
\sum_{n \in D_{m}} \varphi(n) \\
\quad= \begin{cases}q^{m}-1 & \text { if } m \text { is a power of } 2, q \text { is odd, } \\
q^{m} & \text { if } m \text { is a power of } 2, q \text { is even, } \\
\sum_{i \mid m} \mu(i) q^{m / i} & \text { if } m \text { is not a power of } 2 . \\
i \text { odd } & \end{cases}
\end{array}
$$

To prove Proposition 6, we need some arithmetical facts about greatest common divisors.

Lemma 7. Let e, $f, s, m \in \mathbb{N}$. Then

$$
\begin{array}{r}
\operatorname{gcd}\left(q^{e}+1, q^{f}-1\right) \\
= \begin{cases}1, & \text { if } \operatorname{gcd}(2 e, f)=\operatorname{gcd}(e, f), 2 \mid q \\
2, & \text { if } \operatorname{gcd}(2 e, f)=\operatorname{gcd}(e, f), 2 \nmid q \\
q^{\operatorname{gcd}(e, f)}+1, & \text { if } \operatorname{gcd}(2 e, f)=2 \operatorname{gcd}(e, f),\end{cases}
\end{array}
$$

$$
\begin{aligned}
\operatorname{gcd}\left(q^{s}+1, q^{m}+1\right) & \begin{cases}1, & \text { if } v(m) \geq v(m-s), 2 \mid q \\
2, & \text { if } v(m) \geq v(m-s), 2+q \\
q^{\operatorname{gcd}(m, s)}+1, & \text { if } v(m)<v(m-s),\end{cases}
\end{aligned}
$$

where $v(j)=v_{2}(j)$ denotes the highest power of 2 that divides $j \in \mathbb{N}$. 
Proof. To prove (13), we consider two separate cases.

Case $1(\operatorname{gcd}(2 e, f)=\operatorname{gcd}(e, f))$. Observe that

$$
\begin{aligned}
\operatorname{gcd} & \left(q^{e}+1, q^{f}-1\right) \mid \operatorname{gcd}\left(q^{2 e}-1, q^{f}-1\right) \\
= & q^{\operatorname{gcd}(2 e, f)}-1=\left(q^{\operatorname{gcd}(e, f)}-1\right) \mid\left(q^{e}-1\right) .
\end{aligned}
$$

If $2 \mid q$, then $\operatorname{gcd}\left(q^{e}+1, q^{e}-1\right)=1$, and we infer that $\operatorname{gcd}\left(q^{e}+\right.$ $\left.1, q^{f}-1\right)=1$.

If $2+q$, then $\operatorname{gcd}\left(q^{e}+1, q^{e}-1\right)=2$, and we infer that $\operatorname{gcd}\left(q^{e}+1, \mathrm{q}^{f}-1\right)=2$.

Case $2(\operatorname{gcd}(2 e, f)=2 \operatorname{gcd}(e, f))$. Observe that

$$
\begin{aligned}
\operatorname{gcd} & \left(q^{e}+1, q^{f}-1\right) \mid \operatorname{gcd}\left(q^{2 e}-1, q^{f}-1\right) \\
& =q^{\operatorname{gcd}(2 e, f)}-1=q^{2 \operatorname{gcd}(e, f)}-1 \\
& =\left(q^{\operatorname{gcd}(e, f)}-1\right)\left(q^{\operatorname{gcd}(e, f)}+1\right) .
\end{aligned}
$$

If $2 \mid q$, then $\operatorname{gcd}\left(q^{e}+1, q^{\operatorname{gcd}(e, f)}-1\right)=1$, and from (16), we have

$$
\operatorname{gcd}\left(q^{e}+1, q^{f}-1\right) \mid\left(q^{\operatorname{gcd}(e, f)}+1\right) .
$$

On the other hand,

$$
\left(q^{\operatorname{gcd}(e, f)}+1\right)\left|\left(q^{2 \operatorname{gcd}(e, f)}-1\right)=\left(q^{\operatorname{gcd}(2 e, f)}-1\right)\right|\left(q^{f}-1\right) .
$$

Since $e / \operatorname{gcd}(e, f)$ is odd, we have $\left(q^{\operatorname{gcd}(e, f)}+1\right) \mid\left(q^{e}+1\right)$. This last relation together with (17) and (18) yields $\operatorname{gcd}\left(q^{e}+1, q^{f}-\right.$ $1)=q^{\operatorname{gcd}(e, f)}+1$, as required.

If $2 \nmid q$, then $\operatorname{gcd}\left(\left(q^{e}+1\right) / 2,\left(q^{\operatorname{gcd}(e, f)}-1\right) / 2\right)=1$, and so we infer from (16) that

$$
\operatorname{gcd}\left(\frac{q^{e}+1}{2}, \frac{q^{f}-1}{2}\right) \mid\left(q^{\operatorname{gcd}(e, f)}+1\right) .
$$

Since (18) holds, we have

$$
\frac{q^{\operatorname{gcd}(e, f)}+1}{2} \mid \frac{q^{f}-1}{2} .
$$

Since $e / \operatorname{gcd}(e, f)$ is odd, we also have

$$
\frac{q^{\operatorname{gcd}(e, f)}+1}{2} \mid \frac{q^{e}+1}{2} .
$$

The relations (20) and (21) show that

$$
\operatorname{gcd}\left(\frac{q^{e}+1}{2}, \frac{q^{f}-1}{2}\right)=\frac{q^{\operatorname{gcd}(e, f)}+1}{2} \operatorname{gcd}\left(k_{1}, k_{2}\right)
$$

for some $k_{1}, k_{2} \in \mathbb{N}$. Taking (19) into account, we deduce that $\operatorname{gcd}\left(k_{1}, k_{2}\right)=1$ or 2 . To complete the proof, it suffices to verify that $\operatorname{gcd}\left(k_{1}, k_{2}\right)=1$. To this end, suppose that $\operatorname{gcd}\left(k_{1}, k_{2}\right)=2$. Thus, (22) yields

$$
2\left(q^{\operatorname{gcd}(e, f)}+1\right) \mid\left(q^{e}+1\right) .
$$

Since $e / \operatorname{gcd}(e, f)$ is odd, we see that $q^{e}+1=\left(q^{\operatorname{gcd}(e, f)}+1\right) G$, where $G \in \mathbb{N}$ is odd. Combining this last relation with (23), we arrive that $2 \mid G$, which is untenable.

To prove (14), we first observe that

$$
\begin{aligned}
\operatorname{gcd}(2 e, f) & \operatorname{gcd}(e, f) \Longleftrightarrow v(e) \geq v(f), \\
\operatorname{gcd}(2 e, f) & =2 \operatorname{gcd}(e, f) \Longleftrightarrow v(e)<v(f) .
\end{aligned}
$$

Putting $m=e, m-s=f$ in (13), the desired result (14) follows at once if we can show that

$$
\operatorname{gcd}\left(q^{m}+1, q^{m-s}-1\right)=\operatorname{gcd}\left(q^{m}+1, q^{s}+1\right) .
$$

To this end, let $d_{1}=\operatorname{gcd}\left(q^{m}+1, q^{m-s}-1\right), d_{2}=\operatorname{gcd}\left(q^{m}+\right.$ $\left.1, q^{s}+1\right)$. It is easily checked that $d_{1}=d_{2}$ because $d_{2} \mid d_{1} \Leftrightarrow$ $d_{2} \mid\left(q^{m-s}-1\right)$ and $d_{1}\left|d_{2} \Leftrightarrow d_{1}\right|\left(q^{s}+1\right)$.

We are now ready to prove Proposition 6.

Case 1 ( $m$ is a power of 2$)$. Clearly, in this case $\nu(m) \geq v(m-s)$ for $0 \leq s<m$. For brevity, let

$$
\begin{aligned}
S_{m}:= & \left\{n \in \mathbb{N} ; n \mid\left(q^{s}+1\right)\right. \\
& \left.\quad \text { for some } s \in\{0,1, \ldots, m-1\}, n \mid\left(q^{m}+1\right)\right\} .
\end{aligned}
$$

Thus, $\sum_{n \in D_{m}} \varphi(n)=\sum_{n \mid\left(q^{m}+1\right)} \varphi(n)-\sum_{n \in S_{m}} \varphi(n)$. If $2 \mid q$, then using (14), we see that those $s \in\{0,1, \ldots, m-1\}$ for which $n \mid\left(q^{s}+1\right)$ must satisfy $\operatorname{gcd}\left(q^{s}+1, q^{m}+1\right)=1$, showing that $n=1$. On the other hand, if $2+q$, then using (14), we see those $s \in\{0,1, \ldots, m-1\}$ for which $n \mid\left(q^{s}+1\right)$ must satisfy $\operatorname{gcd}\left(q^{s}+1, q^{m}+1\right)=2$, showing that $n=1,2$. Combining the two possibilities, we get

$$
\begin{aligned}
& \sum_{n \in D_{m}} \varphi(n) \\
& \quad=\sum_{n \mid\left(q^{m}+1\right)} \varphi(n)- \begin{cases}\varphi(1), \\
\varphi(1)+\varphi(2), & \text { if } 2+q,\end{cases} \\
& \quad= \begin{cases}q^{m}, & \text { if } 2 \mid q, \\
q^{m}-1, & \text { if } 2+q .\end{cases}
\end{aligned}
$$

Case 2 ( $m$ is not a power of 2$)$. Then $m=2^{v} u$, where $v:=$ $\nu(m)$ and $u(\geq 3)$ is odd. From (14), we need to find those $s \in$ $\{0,1, \ldots, m-1\}$ for which $\nu(m-s)>v(m)$. These are $s$ such that $m-s=2^{v+1} w(w \in \mathbb{N})$; that is, $s=2^{v}(u-2 w)$. Thus, all such $s$ are of the form

$$
s=2^{v} \cdot 1,2^{v} \cdot 3, \ldots, 2^{v} \cdot(u-2)
$$


and correspondingly, $\operatorname{gcd}(s, m)=2^{v} c$, for some $c \mid u, 1 \leq c<$ $u$. We proceed now by induction on $u$, noting that the case $u=1$ (and $u=2$ ) is contained in Case 1 . We have

$$
\begin{aligned}
& \sum_{n \in D_{m}} \varphi(n) \\
& =\sum_{n \mid\left(q^{m}+1\right)} \varphi(n)-\sum_{n \in S_{m}} \varphi(n) \\
& =q^{m}-\sum_{\substack{c \mid u \\
1<c<u}} \sum_{i \mid c} \mu(i) q^{2^{v} c / i}- \begin{cases}0+q^{2^{v}} & \text { if } 2 \mid q \\
1+q^{2^{v}}-1 & \text { if } 2+q\end{cases} \\
& =q^{m}-\sum_{\substack{c \mid u \\
1 \leq c<u}} \sum_{i \mid c} \mu(i) q^{2^{v} c / i} \\
& =q^{m}-\sum_{\substack{k \mid u \\
1 \leq k<u}} q^{2^{v} k} \sum_{\substack{i \mid(u / k) \\
1 \leq i<u / k}} \mu(i) \\
& =q^{m}+\sum_{\substack{k \mid u \\
1 \leq k<u}} q^{2^{v} k} \mu\left(\frac{u}{k}\right)=\sum_{\substack{j \mid m \\
j \text { odd }}} \mu(j) q^{m / j} .
\end{aligned}
$$$$
=q^{m}+1-\sum_{\substack{c \mid u \\ 1 \leq c<u}} \sum_{n \in D_{2^{v}} c} \varphi(n)+ \begin{cases}\varphi(1) & \text { if } 2 \mid q \\ \varphi(1)+\varphi(2) & \text { if } 2+q\end{cases}
$$$$
\text { (using induction) }
$$

\section{Order of a Polynomial}

Following [3, page 84], for $f(x) \in \mathbb{F}_{q}[x] \backslash\{0\}$ with $f(0) \neq 0$, the least $e \in \mathbb{N}$ for which $f(x)$ divides $x^{e}-1$ is called the order of $f(x)$, denoted by ord $(f)$. If $f(0)=0$, then $f(x)=$ $x^{h} g(x)$, where $h \in \mathbb{N}$ and $g \in \mathbb{F}_{q}[x]$ with $g(0) \neq 0$ are uniquely determined, and ord $(f)$ is defined to be ord $(g)$. The order of a polynomial is also called the period or the exponent of that polynomial. The following facts are well-known.

(i) (see [3, Theorem 3.3]) Let $f(x) \in \mathbb{F}_{q}[x]$ be irreducible with $\operatorname{deg}(f)=m \in \mathbb{N}, f(0) \neq 0$. Then ord $(f)$ is equal to the order of any root of $f$ in the multiplicative group $\mathbb{F}_{q^{m}}^{*}$.

(ii) (see [3, Lemma 3.6]) Let $c \in \mathbb{N}$. Then $f \in \mathbb{F}_{q}[x]$ with $f(0) \neq 0$ divides $x^{c}-1$ if and only if $\operatorname{ord}(f) \mid c$.

(iii) (see [3, Theorem 3.8]) Let $g \in \mathbb{F}_{q}[x]$ be irreducible over $\mathbb{F}_{q}$ with $g(0) \neq 0$ and $\operatorname{ord}(g)=e$, and let $f=g^{b}$ with $b \in \mathbb{N}$. Let $t$ be the smallest integer with $p^{t} \geq b$, where $p$ is the characteristic of $\mathbb{F}_{q}$. Then $\operatorname{ord}(f)=e p^{t}$.

(iv) (see [3, Theorem 3.9]) Let $g_{1}, \ldots, g_{k}$ be pairwise relatively prime nonzero polynomials over $\mathbb{F}_{q}$, and let $f=$ $g_{1} \cdots g_{k}$. Then ord $(f)=\operatorname{lcm}\left\{\operatorname{ord}\left(g_{1}\right), \ldots, \operatorname{ord}\left(g_{k}\right)\right\}$.

3.1. Counting Formulae. Working with $q$-cycles, apart from the known result, [3, Theorem 3.5], that the number of monic irreducible $f \in \mathbb{F}_{q}[x], f(0) \neq 0$ for which $\operatorname{ord}(f)=N_{r}(r \in$
$\{1, \ldots, d\})$ and $\operatorname{deg}(f)=o_{N_{r}}(q)$ is $\varphi\left(N_{r}\right) / o_{N_{r}}(q)$, some new information about the number of monic irreducible polynomials is now derived.

Theorem 8. (i) The polynomial $f \in \mathbb{F}_{q}[x]$ constructed via (2) from a $q(a)$-cycle $(N)$ with $\operatorname{gcd}(a, N)=N_{r}(r \in\{1, \ldots, d\})$ is a monic irreducible polynomial with $f(0) \neq 0$, ord $(f)=$ $N / N_{r}$ and $\operatorname{deg}(f)=o_{N / N_{r}}(q)$. Conversely, each irreducible polynomial $f \in \mathbb{F}_{q}[x]$ with $f(0) \neq 0$, ord $(f)=N_{r}$ and $\operatorname{deg}(f)=o_{N_{r}}(q)$ is an irreducible factor of $x^{N}-1$ arisen, through (2), from an $q(a)$-cycle $(N)$ with $\operatorname{gcd}(a, N)=N / N_{r}$.

(ii) The set of monic, irreducible $f \in \mathbb{F}_{q}[x], f(0) \neq 0$ for which ord $(f) \mid N$ is identical with the set of irreducible factors of $x^{N}-1$.

(iii) The number of $q$-cycles $(N)$, the number of monic irreducible factors of $x^{N}-1$, and the number of monic irreducible $f \in \mathbb{F}_{q}[x]$ with $f(0) \neq 0$ and ord $(f) \mid N$ are all equal to $\sum_{r=1}^{d} \varphi\left(N_{r}\right) / o_{N_{r}}(q)$.

Proof. From Theorem 1(v), each $q(a)-\operatorname{cycle}(N)$ with $\operatorname{gcd}$ $(a, N)=N_{r}$ has length $o_{N / N_{r}}(q)$ and it thus gives rise, through the $\alpha$-correspondence, to a monic irreducible $f \in \mathbb{F}_{q}[x]$, $f(0) \neq 0$, of degree $o_{N / N_{r}}(q)$. Each root of $f$ is of the form $\alpha^{a q^{i}}\left(i \in\left\{0,1, \ldots, o_{N / N_{r}}(q)-1\right\}\right)$ where $\alpha$ is a primitive $N$ th root of unity, and so its order is $N / N_{r}$. Part (i) is thus a consequence of [3, Theorem 3.3] and the $\alpha$-correspondence.

To prove (ii), note that if the monic irreducible $f \in \mathbb{F}_{q}[x]$ satisfies $f(0) \neq 0$ and $\operatorname{ord}(f) \mid N$, then ord $(f)=N_{r}$ for some $r \in\{1, \ldots, d\}$. Thus, $f \mid\left(x^{N_{r}}-1\right)$, and so $f \mid\left(x^{N}-1\right)$. On the other hand, if $f$ is an irreducible factor of $x^{N}-1$, the $\alpha$-correspondence assures us that $f$ is constructed from a $q$ $\operatorname{cycle}(N)$ and the desired conclusion follows from (i).

Part (iii) follows (ii), the $\alpha$-correspondence, and Theorem 1(viii).

There is another straightforward technique to derive a formula for the number of monic irreducible polynomials of fixed order using $q$-cycles based on the inclusion-exclusion principle. Although the formula so obtained is not easy to use, we give a proof to illustrate this different approach. For $r \in\{1,2, \ldots, d\}$, set (keeping the earlier notation in the first section)

$$
\begin{aligned}
& \mathscr{I}_{N_{r}}=\{\text { monic irreducible polynomials arising from } \\
& \text { the } \left.q \text {-cycles }\left(N_{r}\right) \text { as constructed in }(2)\right\}, \\
& \qquad \mathcal{O}_{N_{d}}=\mathcal{O}_{1}=\left|\mathscr{C}_{1}\right|, \\
& \mathcal{O}_{N_{r}}=\left|\mathscr{C}_{N_{r}}\right|-\sum_{M \mid N_{r}, M<N_{r}} \mathcal{O}_{M} .
\end{aligned}
$$

For a given $s \in \mathbb{N}$ and distinct primes $p_{1}, p_{2}, \ldots, p_{k}$, define

$$
\begin{gathered}
\langle s\rangle:=\left\{p_{1}^{n_{1}} p_{2}^{n_{2}} \cdots p_{k}^{n_{k}} ; n_{1}, n_{2}, \ldots, n_{k} \in \mathbb{N},\right. \\
\left.n_{1}+n_{2}+\cdots+n_{k}=s\right\} .
\end{gathered}
$$


Proposition 9. Let $N=p_{1}^{n_{1}} p_{2}^{n_{2}} \cdots p_{k}^{n_{k}}$, the unique prime factorization of $N$. The number of monic, irreducible polynomials $f \in \mathbb{F}_{q}[x] \backslash \mathbb{F}_{q}, f(0) \neq 0$ having order $N$ is equal to

$$
\begin{aligned}
& \mathcal{O}_{N}=\left|\mathscr{I}_{N_{1}}\right|-\left|\bigcup_{r=2}^{d} \mathscr{I}_{N_{r}}\right| \\
& =\left|\mathscr{C}_{N_{1}}\right| \\
& -\left\{\sum_{r=2}^{d}\left|\mathscr{C}_{N_{r}}\right|\right. \\
& -\sum_{x_{1}, x_{2} \in\left\{N_{2}, N_{3}, \ldots, N_{d}\right\}, x_{1}<x_{2}}\left|\mathscr{C}_{\operatorname{gcd}\left(x_{1}, x_{2}\right)}\right| \\
& +\cdots+(-1)^{d} \\
& \left.\times \sum_{\substack{x_{1}, x_{2}, \ldots, x_{d-1} \in\left\{N_{2}, N_{3}, \ldots, N_{d}\right\} \\
x_{1}<x_{2}<\cdots<x_{d-1}}}\left|\mathscr{C}_{\operatorname{gcd}\left(x_{1}, x_{2}, \ldots, x_{d-1}\right)}\right|\right\} \\
& =\left|\mathscr{C}_{N_{1}}\right|-\sum_{m \in\langle s-1\rangle}\left|\mathscr{C}_{m}\right| \\
& +\sum_{m \in\langle s-2\rangle}\left|\mathscr{C}_{m}\right|+\cdots+(-1)^{k} \sum_{m \in\langle s-k\rangle}\left|\mathscr{C}_{m}\right| .
\end{aligned}
$$

Proof. Since the number of distinct irreducible factors of $x^{N}-1$ in $\mathbb{F}_{q}[x]$ is equal to the number of $q-\operatorname{cycles}(N)[1$, Corollary 9.12], from its definition, the $\alpha$-correspondence and [3, Lemma 3.6], $\mathcal{O}_{N_{r}}$ is simply the number irreducible factors (in $\mathbb{F}_{q}[x]$ constructed through (2)) of $x^{N_{r}}-1$ which are not irreducible factors of $x^{M}-1$ for any $M \mid N_{r}, M<N_{r}$, which is in turn equivalent to saying that $\mathcal{O}_{N_{r}}$ is the number of monic, irreducible polynomials $f \in \mathbb{F}_{q}[x], f(0) \neq 0$, having order $N_{r}$, and this proves (32).

Again using [1, Corollary 9.12] and [3, Lemma 3.6] to translate the number of $q$-cycles to those of corresponding irreducible polynomials, the inclusion-exclusion formulae on the right-hand sides of (33) and (34) clearly yield the number of irreducible factors of $x^{N}-1$ which are not irreducible factors of $x^{N_{r}}-1$ for all $r \in\{2,3, \ldots, d\}$, and this verifies (33) and (34).

Remark 10. Let us remark that there are no irreducible polynomials in $\mathbb{F}_{q}[x]$ of order $n$ with $\operatorname{gcd}(q, n)>1$. This is seen as follows: for $f(x) \in \mathbb{F}_{q}[x]$ with $f(0) \neq 0$, since ord $(f)$ is the least positive integer for which $f(x)$ divides $x^{\operatorname{ord}(f)}-1$, monic irreducible polynomials of order $n$ are contained in the set of irreducible factors of $x^{n}-1$ in $\mathbb{F}_{q}[x]$. Putting $n=$ $N p^{e}(e \in \mathbb{N}, \operatorname{gcd}(q, N)=1)$, since $x^{n}-1=\left(x^{N}-1\right)^{p^{e}}$, the set of all irreducible factors of $x^{n}-1$ is identical with the set of all irreducible factors of $x^{N}-1$, and so each irreducible factor of $x^{n}-1$ is of order $\leq N<n$.

In contrast to the preceding remark, there always exists a (reducible) polynomial in $\mathbb{F}_{q}[x]$ of order $n$ with $\operatorname{gcd}(q, n)>1$.
This is seen as follows: using the terminology of the last remark, since $\operatorname{gcd}(q, N)=1$, Theorem 8 assures us that there always exists a monic, irreducible polynomial $g(x) \in \mathbb{F}_{q}[x]$, $g(0) \neq 0$, having $\operatorname{ord}(g)=N$. Consequently, Theorem 3.8 in [3] tells us that $\operatorname{ord}\left(g(x)^{p^{e}}\right)=N p^{e}=n$.

Our next task is to compute the number of monic polynomials in $\mathbb{F}_{q}[x]$ (both reducible and irreducible) having order $N$. Let $f(x) \in \mathbb{F}_{q}[x]$ be a factor of the polynomial $x^{N}-1$ with $\operatorname{ord}(f)=N$. Since $\operatorname{gcd}(q, N)=1$, the polynomial $x^{N}-1$ has no multiple factors. Assume that $f(x)$ is decomposed into $k$ distinct monic irreducible factors (in $\mathbb{F}_{q}[x]$ ), say, $f(x)=f_{1}(x) \cdots f_{k}(x)$. Since $\operatorname{ord}(f)=$ $N=\operatorname{lcm}\left\{\operatorname{ord}\left(f_{1}\right), \ldots, \operatorname{ord}\left(f_{k}\right)\right\}$, each $\operatorname{ord}\left(f_{i}\right) \in\{N=$ $\left.N_{1}>N_{2}>\cdots>N_{d}=1\right\}$ (the set of all divisors of $N$ ). Thus, the number of all such $f$ 's is equal to $\sum_{e_{1}+e_{2}+\cdots+e_{d}=k, e_{r} \geq 0, \operatorname{lcm}\left(N_{r} ; e_{r} \neq 0\right)=N}\left(\begin{array}{c}\mathscr{O}_{N_{1}} \\ e_{1}\end{array}\right) \cdots\left(\begin{array}{c}\mathcal{O}_{N_{d}} \\ e_{d}\end{array}\right)$. We have thus proved.

Theorem 11. The total number of monic polynomials (both reducible and irreducible) $f \in \mathbb{F}_{q}[x], f(0) \neq 0$, having order $N$ is

$$
\sum_{\substack{k=1 \\
e_{1}+e_{2}+\cdots+e_{d}=k, e_{r} \geq 0 \\
\operatorname{lcm}\left(N_{r} ; e_{r} \neq 0\right)=N}}^{m}\left(\begin{array}{c}
\mathcal{O}_{N_{1}} \\
e_{1}
\end{array}\right) \ldots\left(\begin{array}{c}
\mathcal{O}_{N_{d}} \\
e_{d}
\end{array}\right), \quad m:=\sum_{r=1}^{d} \mathcal{O}_{N_{r}} .
$$

Having determined the number of polynomials with fixed order, it is natural to find out how many of them have orders dividing a fixed $n \in \mathbb{N}$.

Theorem 12. Let $n=p^{e} N \in \mathbb{N}$ withe $\in \mathbb{N}_{0}$ and $\operatorname{gcd}(q, N)=$ 1. Then the product of all monic irreducible polynomials $f \in$ $\mathbb{F}_{q}[x], f(0) \neq 0$ for which ord $(f) \mid n$ is equal to $x^{N}-1$ and their number is equal to $\left|\mathscr{C}_{N}\right|=\sum_{r=1}^{d} \varphi\left(N_{r}\right) / o_{N_{r}}(q)$.

Proof. Since $x^{n}-1=\left(x^{N}-1\right)^{p^{e}}$, the set of all monic irreducible factors of $x^{N}-1$ is identical with the set of all monic irreducible factors of $x^{n}-1$. From [3, Theorem 3.6], for $f \in \mathbb{F}_{q}[x], f(0) \neq 0$, we know that ord $(f) \mid n$ if and only if $f(x) \mid\left(x^{n}-1\right)$. Thus, the set of all monic irreducible polynomials $f \in \mathbb{F}_{q}[x], f(0) \neq 0$ for which $\operatorname{ord}(f) \mid n$ is identical with the set of all irreducible factors of $x^{n}-1$ which is also equal to the set of all irreducible factors of $x^{N}-1$. Since $x^{N}-1$ has no multiple root, the product of all monic irreducible polynomials $f \in \mathbb{F}_{q}[x], f(0) \neq 0$ for which $\operatorname{ord}(f) \mid n$ is $x^{N}-1$ and the first assertion is established. The second assertion follows immediately from the first assertion and Theorem 8(iv).

3.2. Some Explicit Shapes. Having counted the number of monic irreducible polynomials of fixed order, we proceed to determine their explicit shapes. From [1, Corollary 9.12], we know that the number of irreducible factors of $x^{N}-1$ in $\mathbb{F}_{q}[x]$ is equal to the number of $q-\operatorname{cycles}(N)$ formed by the $N$ numbers $0,1, \ldots, N-1$. To determine explicit shapes of all 
monic, irreducible polynomials $f \in \mathbb{F}_{q}[x], f(0) \neq 0$, having order $N$, we consider the polynomial

$$
f(x)=\left(x-\alpha^{a_{0}}\right)\left(x-\alpha^{a_{1}}\right) \cdots\left(x-\alpha^{a_{\ell-1}}\right) \in \mathbb{F}_{q}[x]
$$

associated with the $q-\operatorname{cycle}(N)\left(a_{0}, a_{1}, \ldots, a_{\ell-1}\right)$, where $\alpha$ is a primitive $N$ th root of unity. By definition, [3, Lemma 3.6] and [1, Theorem 9.11], among all such polynomials, those which do not divide $x^{N_{r}}-1, r \in\{2,3, \ldots, d\}$, are all the sought after polynomials having order $N$.

Although, the procedure just described is satisfactory in principle, in certain cases, more precise shapes can be given. For a positive integer $n$ not divisible by $p$, recall that the $n$th cyclotomic polynomial [3, page 64$]$ is defined as

$$
Q_{n}(x)=\prod_{\substack{i=1 \\ \operatorname{gcd}(i, n)=1}}^{n}\left(x-\zeta^{i}\right)=\prod_{i \mid n}\left(x^{i}-1\right)^{\mu(n / i)} \in \mathbb{F}_{q}[x]
$$

where $\zeta$ is a primitive $n$th root of unity and $\mu$ is the Möbius function. It is known that

$$
x^{N}-1=\prod_{r=1}^{d} Q_{N_{r}}(x) .
$$

Proposition 13. Let $d=d(N)$ be the number of divisors of $N$.

(i) If the number of $q$-cycles $(N)$ is equal to $d$, then in (38) each $Q_{N_{r}}(x)$ is irreducible in $\mathbb{F}_{q}[x]$ and ord $\left(Q_{N_{r}}\right)=$ $N_{r}$. In particular, $Q_{N}(x) \in \mathbb{F}_{q}[x]$ is irreducible and of order $N$. The same conclusion holds if $q$ is a primitive root $\bmod N_{r}$ for all $r \in\{1,2, \ldots, d\}$.

(ii) If $Q_{N_{r}}(x)$ is irreducible in $\mathbb{F}_{q}[x]$ for all $r \in\{1,2, \ldots, d\}$, then the number of $q$-cycles $(N)$ is equal to $d$.

Proof. We prove only part (i), for the other part is trivial from (38). If the number of $q$-cycles $(N)$ is equal to $d$, the $\alpha$ correspondence shows that the number of monic irreducible factors of $x^{N}-1$ is also $d$. The first assertion that each $Q_{N_{r}}(x) \in \mathbb{F}_{q}[x]$ is irreducible follows at once from (38). This is also the case if $q$ is a primitive root $\bmod N_{r}$ for all $r \in\{1,2, \ldots, d\}$ which is a direct consequence of $[3$, Theorem 2.47(ii)].

From (38), we see that $Q_{N_{r}}$ divides $x^{N_{r}}-1$. If $\operatorname{ord}\left(Q_{N_{r}}\right)=$ $j<N_{r}$, then $Q_{N_{r}}$ divides $x^{j}-1$. Further, Lemma 3.6 of [3] shows that $j \mid N$, and so $x^{j}-1$ divides $x^{N}-1$. The first assertion then implies that each cyclotomic factor of $x^{N_{r}}-1$ and of $x^{j}-1$ (as in (38)) is irreducible. Since $\operatorname{gcd}\left(N_{r}, q\right)=1$, the polynomial $x^{N_{r}}-1$ contains no multiple root and so all its irreducible factors are distinct. But the cyclotomic factorization of $x^{j}-1$ (as in (38)) does not contain the irreducible factor $Q_{N_{r}}$ (because $N_{r}+j$ ), which is a contradiction.

Our next proposition, which shows how the number of $q$-cycles can be used to generate irreducible polynomials, is based on the following known results.

(i) (Berlekamp's factorization theorem, [3, Theorem 4.1]). If $f \in \mathbb{F}_{q}[x]$ is monic and $h \in \mathbb{F}_{q}[x]$ is such that $h^{q} \equiv h \bmod f$, then $f(x)=\prod_{c \in \mathbb{F}_{q}} \operatorname{gcd}(f(x), h(x)-c)$. (ii) (see [1, Theorem 9.13]) For each $q$-cycle $(N)\left(a_{0}, a_{1}\right.$, $\left.\ldots, a_{\ell-1}\right)$, if $g(x)=x^{a_{0}}+x^{a_{1}}+\cdots+x^{a_{\ell-1}}$, then $g(x)^{q} \equiv$ $g(x) \bmod \left(x^{N}-1\right)$.

Combining these two results, we get the following.

Proposition 14. Let $\left(a_{0}, a_{1}, \ldots, a_{\ell-1}\right)$ be a $q$-cycle $(N)$, and $g(x)=x^{a_{0}}+x^{a_{1}}+\cdots+x^{a_{\ell-1}}$. If the number of $q$-cycles $(N)$ is equal to $q$, and if $\operatorname{gcd}\left(x^{N}-1, g(x)-c\right) \neq 1$ for all $c \in \mathbb{F}_{q}$, then each polynomial $\operatorname{gcd}\left(x^{N}-1, g(x)-c\right)$ is irreducible over $\mathbb{F}_{q}$.

\section{Degree of a Polynomial}

We begin by listing (without proofs) known facts involving degrees of irreducible polynomials, which can be proved using $q$-cycles.

(i) (see [3, Theorem 3.20]) The product of all monic irreducible $f \in \mathbb{F}_{q}[x], f(0) \neq 0$ for which $\operatorname{deg}(f) \mid n$ is equal to $x^{q^{n}-1}-1$.

(ii) (see [3, Theorem 3.25]) The number of monic irreducible polynomials in $\mathbb{F}_{q}[x]$ of degree $\ell$ is equal to $(1 / \ell) \sum_{k \mid \ell} \mu(k) q^{\ell / k}$

(iii) (see [3, Corollary 3.4]) The set of monic irreducible $f \in \mathbb{F}_{q}[x], f(0) \neq 0$ for which $\operatorname{deg}(f) \mid n$ is identical with the set of monic irreducible $f \in \mathbb{F}_{q}[x], f(0) \neq 0$ for which ord $(f) \mid\left(q^{n}-1\right)$. Moreover, for $f \in \mathbb{F}_{q}[x]$, $f(0) \neq 0$, we have $\operatorname{deg}(f) \mid n$ if and only if $\operatorname{ord}(f) \mid$ $\left(q^{n}-1\right)$.

Using $q$-cycles, that is, Theorem 1 (viii) and the assertion [3, Theorem 3.20] the following new result is immediate.

Theorem 15. The number of monic irreducible polynomials $f(x) \in \mathbb{F}_{q}[x], f(0) \neq 0$ for which $\operatorname{deg}(f) \mid n$ is equal to $\left|\mathscr{C}_{q^{n}-1}\right|=\sum_{M \mid q^{n-1}} \varphi(M) / o_{M}(q)$, where the definition of $\mathscr{C}_{q^{n}-1}$ can be found in the heading "Notation and Terminology".

Combining Theorems 15 and 2(iii), we obtain a nice arithmetical identity.

Corollary 16. One has $\sum_{M \mid\left(q^{n}-1\right)}\left(\varphi(M) / o_{M}(q)\right)=$ $\sum_{M \mid n}\left((1 / M) \sum_{k \mid M} \mu(k) q^{M / k}\right)-1$.

\section{Coefficients and $q$-Cycle}

Since a given $q$-cycle gives rise, through the $\alpha$-correspondence, to a unique polynomial, we illustrate now how to uniquely determine all coefficients of its corresponding polynomial (2) and conversely.

Theorem 17. Let $\alpha$ be a primitive Nth root of unity, let $m$ be the order of $q \bmod N$, and let $\xi$ be a generator of $\mathbb{F}_{q^{m}}$.

(i) If the $q\left(a_{0}\right)$-cycle $(N)\left(a_{0}, a_{1}, \ldots, a_{\ell-1}\right)$ is given, then the coefficients of its corresponding polynomial in (2) 


$$
\begin{aligned}
& h(x)=\left(x-\alpha^{a_{0}}\right)\left(x-\alpha^{a_{1}}\right) \cdots\left(x-\alpha^{a_{\ell-1}}\right):=\sum_{k=0}^{\ell}(-1)^{k} \\
& c_{k} x^{\ell-k} \in \mathbb{F}_{q}[x] \text { are given by } \\
& c_{0}=1, \\
& c_{k}=\sum_{0 \leq i_{0}<i_{1}<\cdots<i_{k-1} \leq \ell-1} \alpha^{a_{i_{0}}} \alpha^{a_{i_{1}}} \cdots \alpha^{a_{i_{k-1}}} \quad(k=1, \ldots, \ell) .
\end{aligned}
$$

(ii) If the coefficients of $(x)$ are given, then the corresponding q-cycle is uniquely determined through its leading element which is found by solving for $s, A$, and $a_{0}$, respectively, from the system

$$
\begin{gathered}
\xi^{s}=(-1)^{\ell} c_{\ell}, \\
\left(\frac{q^{m}-1}{N}\right) A \equiv s \bmod q^{m}-1, \\
a_{0}\left(1+q+\cdots+q^{\ell-1}\right) \equiv A \bmod N .
\end{gathered}
$$

Proof. (i) The relations in (39) are simply the symmetric polynomial relations between the roots and coefficients of a polynomial.

(ii) It is trivial that each $q$-cycle is uniquely determined from its leading element. Next, note that (40) is simply a representation of element in $\mathbb{F}_{q^{m}}$. Through the $\alpha$ correspondence, the $q$-cycle $\left(a_{0}, a_{1}, \ldots, a_{\ell-1}\right)$ associated with $h(x)$ must satisfy

$$
\begin{gathered}
\xi^{s}=(-1)^{\ell} c_{\ell}=\alpha^{t}, \\
t:=a_{0}+a_{1}+\cdots+a_{\ell-1} \\
\equiv a_{0}\left(1+q+\cdots+q^{\ell-1}\right) \bmod N .
\end{gathered}
$$

The conclusion follows from the facts that $\alpha=\xi^{\left(q^{m}-1\right) / N}$ and that solving (41) and (42) yields $a_{0}$ uniquely $\bmod N$.

\section{Primitive and Self-Reciprocal Polynomials}

We now apply preceding results to two special cases, corresponding to $N=q^{n}-1$ and $N=q^{m}+1$, to deduce a number of results about primitive and self-reciprocal polynomials.

6.1. Primitive Polynomials. Recall from [3, Definition 3.15] that a primitive polynomial of degree $n \in \mathbb{N}$ over $\mathbb{F}_{q}$ is the minimal polynomial over $\mathbb{F}_{q}$ of a primitive element of $\mathbb{E}_{q^{n}}$. We mention, without proofs, some known results provable using q-cycles.

(1) (see [3, Theorem 3.16]) An irreducible polynomial $f(x) \in \mathbb{F}_{q}[x]$ of degree $n$ is primitive if and only if $f$ is monic, $f(0) \neq 0$ and $\operatorname{ord}(f)=q^{n}-1$. In other words, each primitive polynomial in $\mathbb{F}_{q}[x]$ of degree $n$ can be constructed from a $q(a)$-cycle $\left(q^{n}-1\right)$ with $\operatorname{gcd}\left(a, q^{n}-1\right)=1$ through (2). Conversely, for each $q(a)$-cycle $\left(q^{n}-1\right)$ with $\operatorname{gcd}\left(a, q^{n}-1\right)=1$, the polynomial (2) is a primitive polynomial in $\mathbb{F}_{q}[x]$ of degree $n$.
(2) (see [1, Theorem 7.7]) The number of primitive polynomials in $\mathbb{F}_{q}[x]$ of degree $n$ is $\varphi\left(q^{n}-1\right) / n$, which equals to the number of $q(a)-\operatorname{cycle}\left(q^{n}-1\right)$ with $\operatorname{gcd}\left(a, q^{n}-1\right)=1$.

6.2. Self-Reciprocal Polynomials. Let $f(x)=a_{n} x^{n}+a_{n-1} x^{n-1}+$ $\cdots+a_{1} x+a_{0} \in \mathbb{F}_{q}[x]$ with $a_{n} \neq 0$. The reciprocal polynomial $f^{*}$ of $f$ is defined by $f^{*}(x)=x^{n} f(1 / x)=a_{0} x^{n}+a_{1} x^{n-1}+$ $\cdots+a_{n-1} x+a_{n}$. A nonzero polynomial $f \in \mathbb{F}_{q}[x]$ is called selfreciprocal if $f(x)=f^{*}(x):=x^{n} f(1 / x)$. We confine ourselves here to the study of self-reciprocal irreducible monic (srim) polynomials in $\mathbb{F}_{q}[x]$. Since there is only one first degree srim-polynomial, namely, $x+1$, throughout the rest of this section we treat only srim-polynomials of degree $\geq 2$. We next mention a known characterization and results about srimpolynomials provable via $q$-cycles.

(i) (see [2, page 275]) Let $f \in \mathbb{F}_{q}[x]$ be irreducible and monic of degree $\geq 2$. Then $f$ is self-reciprocal if and only if its set of roots (each of which is evidently nonzero) is closed under inversion (and so its degree must be even).

(ii) (see [2, Proposition 3]) If $f \in \mathbb{F}_{q}[x]$ is a srimpolynomial with $\operatorname{deg}(f)=2 m$, then $\operatorname{ord}(f) \mid\left(q^{m}+1\right)$ but $\operatorname{ord}(f)+\left(q^{k}+1\right)$ for all $k \in\{0,1, \ldots, m-1\}$.

(iii) (see [4, Lemma 2.3], [5, Theorem 1(i)], [2, Corollary 5]) For $a \in \mathbb{Z}_{q^{m}+1}$ with $\operatorname{gcd}\left(a, q^{m}+1\right)=N_{r}(r \in$ $\{1, \ldots, d\})$, each $q(a)$-cycle $\left(q^{m}+1\right)$, which is of length $o_{N / N_{r}}(q)=2 m$, gives rise through (2) to a srimpolynomial in $\mathbb{F}_{q}[x]$ of degree $2 m$ and order dividing $q^{m}+1$. Conversely, each srim-polynomial $f \in \mathbb{F}_{q}[x]$ of degree $2 m$ arises via (2) from a $q(a)$-cycle $\left(q^{m}+1\right)$ with length $o_{\left(q^{m}+1\right) / N_{r}}(q)=2 m$ and $\operatorname{ord}(f) \mid\left(q^{m}+1\right)$, where $N_{r}=\operatorname{gcd}\left(a, q^{m}+1\right)$.

(iv) (see [5, Theorem 1(ii)]) Each irreducible factor $f(x)$ of $x^{q^{m}+1}-1$ with $\operatorname{deg}(f) \geq 2$ is a srim-polynomial with $\operatorname{deg}(f)=2 k$, where $k \mid m$ and $m / k$ is odd.

We next prove two new results using $q$-cycles.

Theorem 18. (i) For each divisor $N_{r}$ of $q^{m}+1$, the number of all srim-polynomial's $f \in \mathbb{F}_{q}[x]$ for which ord $(f)=N_{r}$ and $\operatorname{deg}(f)=o_{N_{r}}(q)$ is $\varphi\left(N_{r}\right) / o_{N_{r}}(q)$.

(ii) The number of srim-polynomials of degree $2 m$ in $\mathbb{F}_{q}[x]$ is equal to $(1 / 2 m) \sum_{1 \leq r \leq d, o_{N_{r}}(q)=2 m} \varphi\left(N_{r}\right)$.

Proof. (i) and (ii) follow from [4, Lemma 2.3], [5, Theorem 1(ii)], Theorem 4, [3, Theorem 3.5], and Theorem 8(ii).

Combining [2, Proposition 3], [5, Theorem 1(i)-(ii)], and Theorem 18 with results in Section 2, we obtain the following known results about the set $D_{m}$ as defined in (11).

(i) (see [2, Proposition 4]) Assume that $f$ is a srimpolynomial over $\mathbb{F}_{q}$ of degree $2 m$ and let $\alpha \in \mathbb{F}_{q^{2 m}}$ be a root of $f$. Then $\alpha$ is a primitive $D$ th root of unity for some $D \in D_{m}$. 
(ii) (see [3, Exercise 3.15, page 141]) If $f$ is a srimpolynomial in $\mathbb{F}_{q}[x]$ of degree $>1$ and order $e$, then every monic irreducible polynomial in $\mathbb{F}_{q}[x]$ of degree $>1$ whose order divides $e$ is self-reciprocal.

(iii) (see $[2$, Corollary 5], [6, Theorem 2]) If $f$ is a srimpolynomial of degree $2 m$ over $\mathbb{F}_{q}$, then ord $(f) \in D_{m}$.

(iv) (see [2, Proposition 7]) Let $D \in D_{m}$ and let $\beta$ be a primitive $D$ th root of unity. If $f_{\beta}(x)=\prod_{i=0}^{2 m-1}(x-$ $\beta^{q^{i}}$, then the polynomial $f_{\beta}$ is an srim-polynomial of degree $2 m$ and order $D$.

(v) (see [2, Theorem 8]) For a monic irreducible polynomial $f$ of degree $2 m$ over $\mathbb{F}_{q}$, the following statements are equivalent.

(1) $f$ is self-reciprocal.

(2) $\operatorname{ord}(f) \in D_{m}$.

(3) $f=f_{\beta}$ for some primitive $D$ th root of unity $\beta$, with $D \in D_{m}$.

(vi) (see [2, Theorem 9])

(1) There are $\varphi(D) / 2 m$ srim-polynomials in $\mathbb{F}_{q}[x]$ of degree $2 m$ and order $D$ for each $D \in D_{m}$.

(2) The number of srim-polynomials in $\mathbb{F}_{q}[x]$ of degree $2 m$ is $(1 / 2 m) \sum_{D \in D_{m}} \varphi(D)$.

(vii) (see [2, Theorem 11]) For $D \in D_{m}$, the $D$ th cyclotomic polynomial $Q_{D}$ factors into the product of all srimpolynomials over $\mathbb{F}_{q}$ of degree $2 m$ and order $D$.

(viii) (see [5, Theorem 3]) Let $S_{q}(m)$ denote the number of srim-polynomials of degree $2 m$ over $\mathbb{F}_{q}$. Then

$$
S_{q}(m)= \begin{cases}\frac{q^{m}-1}{2 m}, & \text { if } q \text { is odd, } m=2^{s} \\ \frac{1}{2 m} \sum_{\substack{d \mid m \\ d \text { odd }}} \mu(d) q^{m / d}, & \text { otherwise. }\end{cases}
$$

\section{Conflict of Interests}

The authors declare that there is no conflict of interests regarding the publication of this paper.

\section{Acknowledgment}

Supported by the Centre of Excellence in Mathematics, the Commission on Higher Education, Thailand.

\section{References}

[1] Z.-X. Wan, Lectures on Finite Fields and Galois Rings, World Scientific, Singapore, 2003.

[2] J. L. Yucas and G. L. Mullen, "Self-reciprocal irreducible polynomials over finite fields," Designs, Codes and Cryptography, vol. 33 , no. 3, pp. 275-281, 2004.
[3] R. Lidl and H. Niederreiter, Finite Fields, Cambridge University Press, Cambridge, UK, 1997.

[4] S. D. Cohen, "Polynomials over finite fields with large order and level," Bulletin of the Korean Mathematical Society, vol. 24, no. 2, pp. 83-96, 1987.

[5] H. Meyn, "On the construction of irreducible self-reciprocal polynomials over finite fields," Applicable Algebra in Engineering, Communication and Computing, vol. 1, no. 1, pp. 43-53, 1990.

[6] O. Ahmadi, "Self-reciprocal irreducible pentanomials over $\mathbb{F}_{2}$," Designs, Codes and Cryptography., vol. 38, no. 3, pp. 395-397, 2006. 


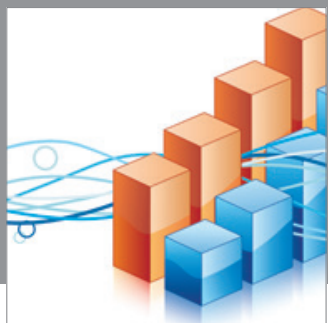

Advances in

Operations Research

mansans

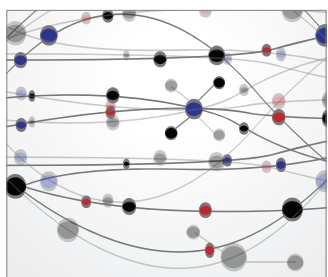

The Scientific World Journal
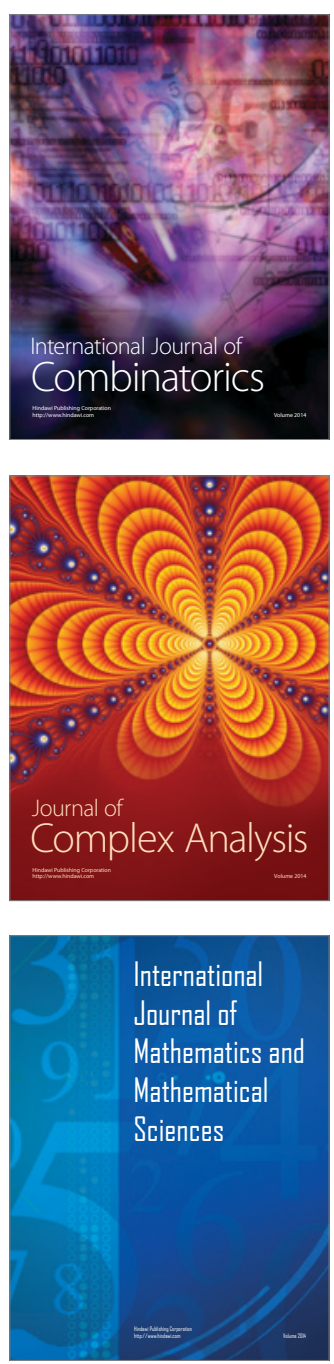
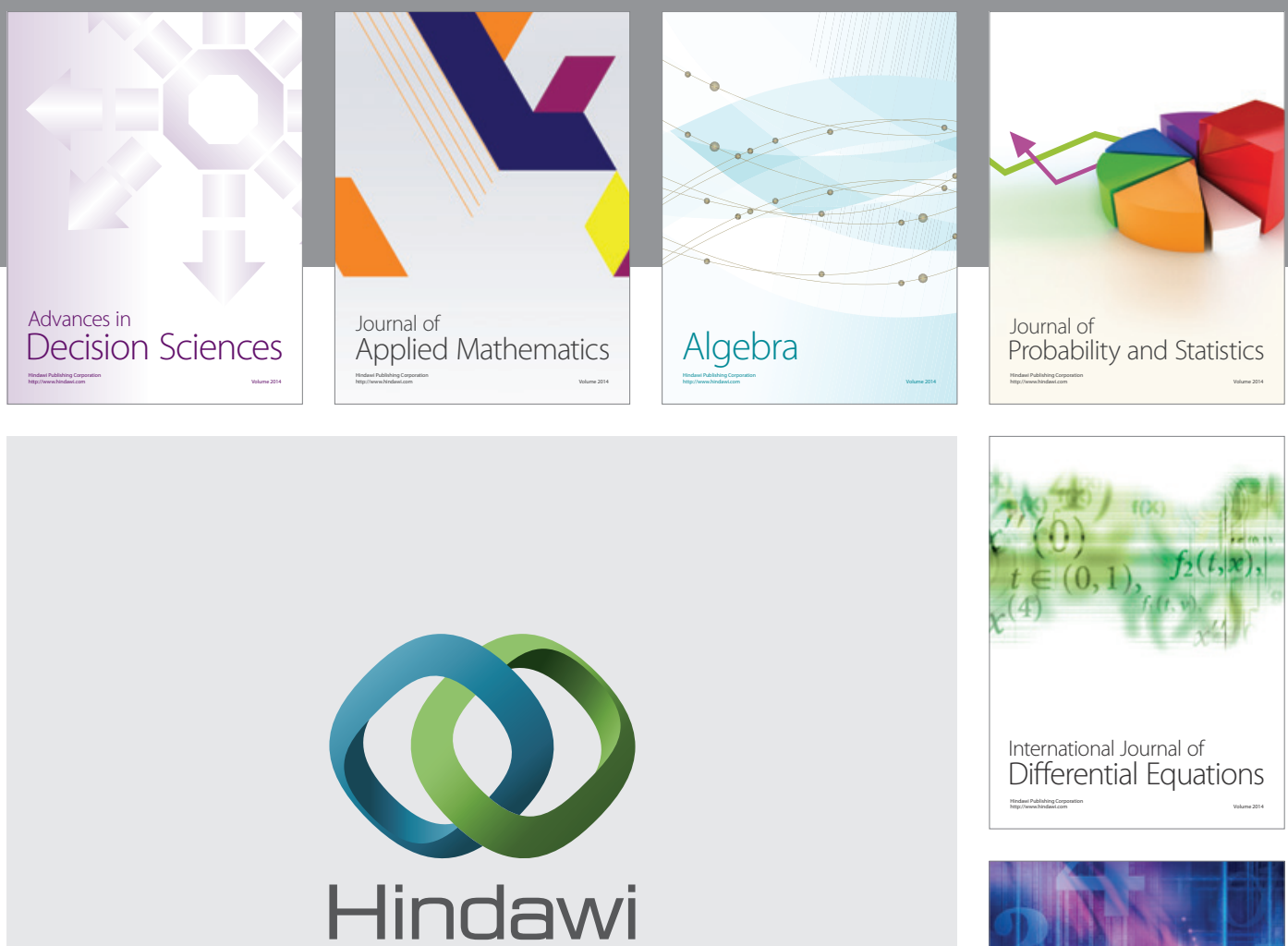

Submit your manuscripts at http://www.hindawi.com
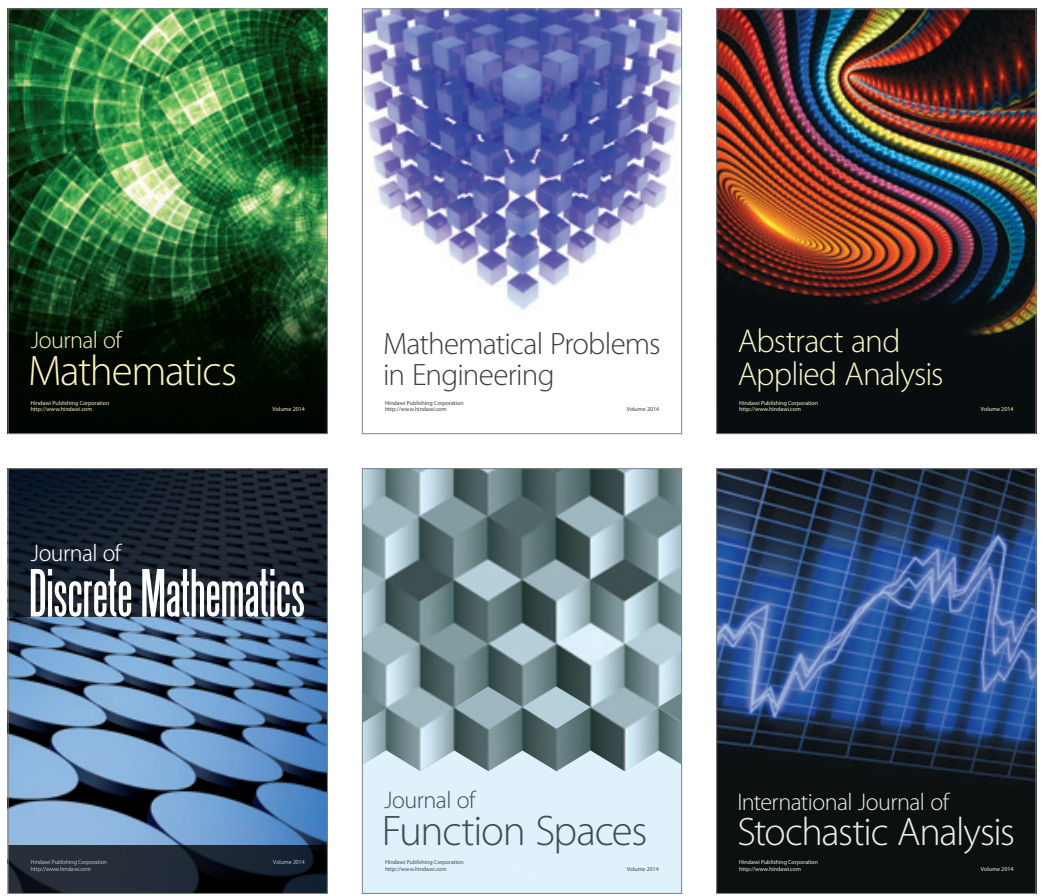

Journal of

Function Spaces

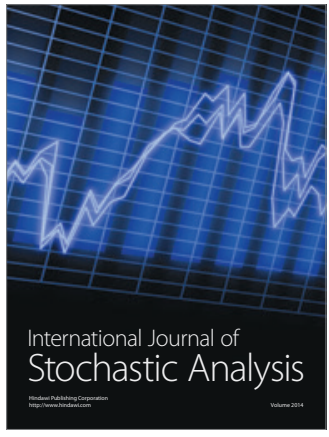

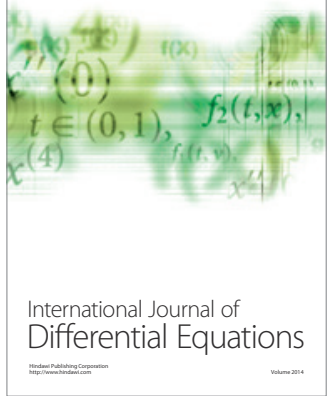
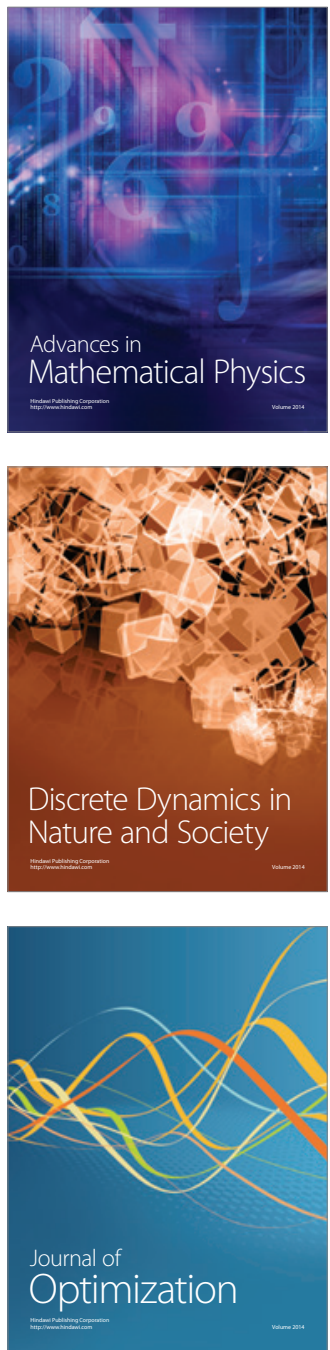